\title{
The social determinants of emerging infectious diseases in Africa
}

\begin{abstract}
The Ebola virus disease (EVD) epidemic in West Africa, from December 6, 2013 to March 2015, was the largest in history. Neither the virus (pathological cause) nor the host (in terms of biological determinants) is the key to understanding the causes or explaining the magnitude and seriousness of the West Africa EVD epidemic. Such a difference in the manifestation of the EVD epidemic in West Africa invites many questions that we would like to explore under the umbrella of "the social determinants of emerging infectious diseases in Africa'. The purpose is to consider these root causes that could explain the actual magnitude in order to address them systematically in dealing with EVD epidemics. We explored the international literature and retrieved the essential contextual root causes of West Africa EVD epidemic before concluding with some areas of action to prevent and addressing such a disease.
\end{abstract}

Volume 8 Issue 2 - 2019

\author{
David Houéto \\ University of Parakou, West Africa
}

Correspondence: David Houéto, School of public health, university of Parakou, BP I23, Parakou, Benin, West Africa, Tel +229972775 15, Email dhoueto@gmail.com

Received: February 23, 2019 | Published: March 12, 2019

Keywords: emerging infectious diseases, ebola, social determinants of health, West Africa

Abbreviations: EVD, ebola virus disease; EBOV, ebolavirus; DRC, democratic republic of congo; EIDs, emerging infectious diseases

\section{Introduction}

The Ebola virus disease (EVD) epidemic in central Africa emerged in West Africa, from December 6, 2013 to March 2015 and has been describes as the largest EVD outbreak in history. ${ }^{1}$ The mainly effected countries were Guinea, Liberia, and Sierra Leone. It is estimated that about 28,600 individuals were infected and that virus claimed over 11,000 lives. ${ }^{2}$ It was the first documented Ebola outbreak in West Africa apart from the Central Africa's series. Although the circulating virus has been identified as the Zaire ebolavirus (EBOV), a strain previously found in only three Central African countries, the Democratic Republic of Congo (DRC), Republic of Congo, and Gabon, the West Africa epidemic was unique in its size, duration, and spatial extent. ${ }^{3}$ Also, the public health impact of the West Africa Ebola epidemic has been far greater than case counts. Neither the virus (pathological cause) nor the host (in terms of biological determinants) are the key to understanding the causes or explaining the magnitude and seriousness of the West Africa Ebola epidemic. ${ }^{4}$ The difference in the manifestation of the EVD epidemic in West Africa invites many questions, notably: why did it occur at that period in time? Why in West Africa? This paper seeks to provide answers to these questions. The interest in finding answers to these questions lies in our quest to understand how social determinants of emerging infectious diseases (EIDs) could inform the design of interventions aiming at controlling outbreaks of emerging infectious diseases in Africa.

\section{Social determinants of health}

According to CSDH/WHO, ${ }^{5}$ social determinants of health (SDH) are the conditions in which people are born, grow, live, work, and age, including the healthcare system. For any population health intervention to be effective, it should aim at changing undesirable elements in the social context that impact on health. ${ }^{6}$ Such undesirable elements would include, without necessarily being limited to, unfair and avoidable or remediable differences in health among groups whether defined socially, economically, demographically or geographically.
SDH have been grouped into three broad categories: behavioral practices, environments (physical and social) factors, and factors related to the health system. As shown in Figure 1, SDH are fueled by inequities ${ }^{7}$ such as socioeconomic position, social structure, social class, education, occupation, income, gender, etc. In broader contexts (national level) these inequities include socioeconomic and political advantages or deprivation relating to governance, macroeconomic policies, and socio-cultural organization of society. This means that, for one to be healthy there must be numerous factors fulfilled in terms of SDH and health equity as shown in Figure 1. Socioeconomic and political context (social environmental category) has a significant contribution to make towards the health status of not only individuals but communities. It is the root cause of health and health equity.

According to Kickbusch ${ }^{8}$ public health professionals need to become more politically astute to achieve their goals, because healthcare has become political, and politics is often the continuous struggle for power among competing interests. Looking at health through the lens of political determinants means analyzing how different power constellations, institutions, processes, interests, and ideological positions affect health within different political systems and cultures and at different levels of governance. She provides three arguments for why health is political. First, health is unevenly distributed, as many health determinants are dependent on political action, and health is a critical dimension of human rights and citizenship. Second, the political cause of health remains the focus of many civil society organizations in global health. Third, the WHO Commission on Social Determinants of Health in its 2008 final report also concluded with the political message that health is shaped ultimately by factors such as: "the distribution of money, power and resources at global, national and local levels"- all of which can be tackled only in sectors other than health.

$\mathrm{Kickbusch}^{8}$ continues by noting that there is currently a renewed politicization of health at all levels of governance, from the local to the global-within governments, global institutions, and the private sector, and through civil society organizations. This means that politics does not just take place in government through elite politicians, but that it permeates society and encompasses "all the processes of conflict, cooperation and negotiation in taking decisions about how 
resources are to be owned, used, produced and distributed." Health has increasing relevance for political legitimacy and the economy, it is critical to fiscal policies and to the social state. That means it affects the interests of many stakeholders and society at large. The politics of the 2014-2015 Ebola outbreak in West Africa provides further illustration of how political determinants shape the responses to outbreaks. How do these considerations relate to EIDs?

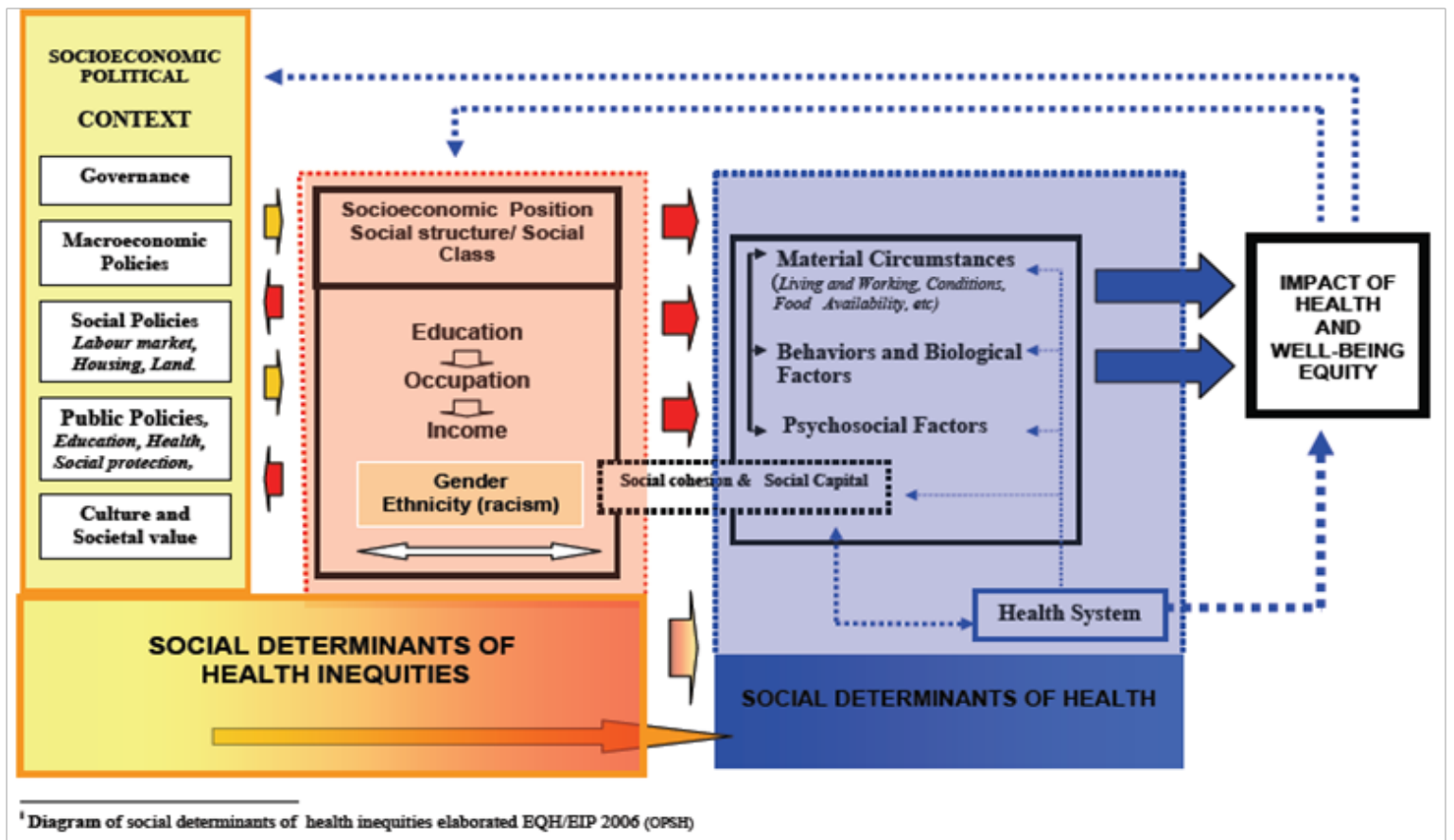

Figure I Conceptual framework corresponding to the determinants of health social inequalities. ${ }^{7}$

\section{Social determinants of EIDs}

If we take the Ebola virus disease (EVD) as an example of an EID, it has registered numerous outbreaks, the majority of them in central Africa, since the first human outbreaks were detected in 1976 in the Democratic Republic of Congo and South Sudan. The first outbreaks had much fewer cases that appear to have ended spontaneously in the general population with limited generation of cases, than subsequent outbreaks. However, when the disease erupted in healthcare settings with low standards of hygiene and sanitation, transmission tended to be amplified and to result in a high number of cases and deaths of healthcare workers. The West African EVD epidemic was a mixture of nosocomial and general population factors, with aggravated human-to-human transmission owing to various reasons including burial practices, inadequate infection control, population density and mobility, cultural beliefs and practices, and fear. ${ }^{9}$

\section{The transmission process}

At the time of the first known outbreak of EVD in the DRC in 1976, it was thought that EVD was primarily transmitted via blood. However, with subsequent EVD outbreaks, increasingly became clear that wasn't the case and that several conditions at local hospitals such as: the lack of running water; limited supply of face masks, caps and gowns; infrequent sterilization of hospital instruments; direct physical contact with EVD patients (both at home and in hospital) were possible means of transmission. Over the years, this list has grown to include: a) Contact with body fluids of an EVD patient, b) sharing a bed during late illness, and in late stage of the disease and c) sharing a meal. Moreover, touching of corpses of persons who had died of EVD have also been identified as a risk factor. ${ }^{10}$ In other words, the risk factors for EVD infection include: exposure to contaminated medical instrument; intimate contact associated with nursing care; direct physical contact with an ill family member (at home or in hospital); and contact with body fluids of an ill person. Also, exposure during late illness constitutes a risk, in cases such as: sharing a meal, conversation, sharing a bed, ritual hand washing during funeral, communal meal during funerals; and contact with the blood of an infected mammal or eating of bush-meat. When analyzing the risk factors above, one can understand that social vulnerability was qualitatively and positively associated with the EVD transmission. The review of the currently available evidence does not reveal any known natural resistance for EBOV infection in humans. ${ }^{11}$ We can distinguish from these risk factors three groups or categories, which are: behavioral practices, environments (physical and social), and the health system.

\section{Behavioral and cultural practices}

A proper understanding of the effects of cultural, traditional and religious practices on the spread of Ebola in West Africa could lead to a strategic shift in efforts to manage the spread of the disease, in the case of an outbreak and in understanding transmission dynamics. The Ebola virus, because of its methods of transmission, is particularly influenced by socio-cultural and behavioral practices that occur at the household and community as well as hospital setting levels. Consequently, the "community" concerned needs always to be defined for the purpose of the public health response. Scientificallybased methods of combatting the spread of highly infectious diseases like Ebola are normally preferred, with very little considerations given to the impact of traditional and religious practices on preventive 
measures. ${ }^{12}$ However, the 2014-2015 trends in EVD outbreaks in West Africa have demonstrated that the use of scientific methods alone, without consideration of other contextual factors, is not sufficient to control the disease. For example, in that 2014-2015 West African EVD epidemic there was notable resistance against prescribed scientific ways of combating the transmission in some affected communities. ${ }^{13}$ While there is no single explanation for this kind of resistance, given its complexity, the influence of religious and sociocultural beliefs cannot be underestimated in the fight against emerging infectious diseases. Also, ethnic and cultural diversity across and within communities further complicates the issue. ${ }^{14}$ Understanding these ethno-social dynamics will help policy-makers in formulating disease prevention approaches that are culturally appropriate during periods of outbreaks. Ebola transmission prevention programs require flexibility and adaptability by governments, public health officials and communities. $^{12}$

\section{Perceived causation of diseases and death}

Adherence to recommended preventive measures is central to the fight against EIDs. However, limited understanding of the etiology of the disease can negatively impact on preventive measures. In an epidemic situation, it is important to note that diseases and death might generally be perceived to have natural and metaphysical causes. Metaphysical causes such as witchcraft entail the spiritual realm and may be seen to be some form of punishment from God or ancestral spirits. ${ }^{15,16}$ This line of thinking can make it difficult for members of affected communities to fully understand the scientific causes of EIDsrelated deaths. It is important to note that West Africa is home to some of the world's fastest-growing Islamic and Christian communities and that the way people conceptualize the etiology of a disease can be linked to their religious beliefs. It is therefore not surprising that during infectious disease outbreaks, it is common for people in such settings to consult or seek help from traditional and spiritual healers. This is often accompanied by rituals aimed at appeasing the gods and ancestors. ${ }^{17}$ In EVD outbreaks, it is common for affected communities to ascribe the cause of the disease to evil spirits, witchcraft, or sorcery, just as it is the case for many other diseases like malaria in the region. ${ }^{16}$ This led many to go to traditional or spiritual healers as the first step in the care seeking itinerary. Traditional or spiritual healers in turn use unsafe practices to treat patients which often lead to complications that may not be easily managed by healthcare facilities. The outcome being an increase in death rates and a resultant inertia by communities to seek care at public health facilities. ${ }^{16}$ More so, when they do, the chances of destruction of treatment units increases and physical attacks on containment teams increases. Moreover, high disease transmission rates in hospitals has been a major cause of mistrust between health care health workers, hospitals and the community at large. It is therefore not surprising that during EVD outbreaks traditional treatment homes often serve as epicenters for disease propagation. ${ }^{18}$

\section{Funeral and burial practices}

Most communities in the West Africa region believe in life after death. Consequently, funeral and burial practices are perceived as crucial steps in transitioning from the world of the living to the spiritual world. It is believed to be a duty for surviving relatives of a deceased person to facilitate the transition from this world to the spiritual world through funeral and burial rituals. Failure to perform this duty which in effect settles the departed relative as an ancestral spirit could result in the deceased coming back from the world beyond to harass and punish surviving relatives. One of the most commonly performed funeral rituals, which significantly contributes to the spread of Ebola, is the washing and cleaning of the corpse followed by washing of hands in a common bowl and then lovingly touching the face of the deceased to cement the bond between the living and the dead. Should the dead happen to be a prominent or endowed person like an artist or traditional healer, it is even common for people to lie over the corpse in the belief that his/her spiritual gifts or endowments might thus be transferred to them. ${ }^{19}$ Given that the major means of human-to-human transmission of EVD is through direct contact with infected body fluids, the afore-mentioned funeral and burial practices inadvertently result in spreading of the disease..$^{15}$ Traditional burial practices, involving washing and touching of the deceased, have been linked to $60 \%$ of Ebola cases in Guinea. ${ }^{20}$ Care giving, primarily by women, has also been associated with outbreaks, presumably indicating a relatively high rate of infection in women $(67 \%$ of affected individuals) in the 2000-2001 Ugandan outbreak. ${ }^{21}$ In spite of the obvious negative impact of burial practices on the spread of Ebola, most West African communities continue to place a significant value on the rituals and resist adoption of alternative methods such as cremation that could minimize the spread of the virus. Against this background, the questions that arise when outbreaks of EIDs occur are the following: How can people be made to desist from practices of getting into contact with corpses? How may a proper burial in line with cultural norms and beliefs be assured while at the same time minimizing the chances of contracting the disease? Is it possible completely to abandon cultural burial practices and enforce scientifically prescribed methods that presumably reduce the spread of the disease?

The WHO, in conjunction with affected governments, had set guidelines defining how bodies of Ebola victims are to be handled and subsequently buried. ${ }^{22}$ But, while the guidelines were formatted in line with current scientific knowledge of the disease, not much attention was given to the socio-cultural implications of some of the prescribed measures. Some of the measures, such as cremation, are in direct conflict with widely held views about life after death. Failure to adopt these measures may sound amazing to people in other cultures. But it is also important to realize that some of these funeral practices span across generations and have been practiced in these communities for centuries and can hardly be changed overnight. For these reasons, some 'Dead Body Management Teams', often carried out burials prior to notifying relatives of the deceased, which created considerable suspicion and discontentment amongst affected communities. As a result, in attempt to avoid having their relatives cremated or buried without their knowledge and consent, some of the people refrained from reporting death in their families and secretly continued with traditional burial practices and, in the process, thwarted efforts to combat the spread of Ebola. Although WHO guidelines do emphasize the need for understanding and respecting cultural practices and religious beliefs, the major challenge is for health officers to convince involved communities to desist from inherent cultural practices. ${ }^{23}$ To address these issues, cultural paradigms must be incorporated into EIDs preparedness plans. As seen in Uganda, some of these paradigms contain elements, such as isolation of cases, restrictions on social gatherings, and safe burial practices, which are consistent with modern principles of EIDs control. Aligning such containment strategies to existing helpful cultural paradigms has proved useful in controlling deadly epidemics. ${ }^{12}$ 


\section{Traditional healers in the care seeking itinerary in West Africa}

Africa has a long history of use of traditional medicine in the management of diseases and illness. It is common in many west African communities for sick persons and/or their families to first consult a traditional healers (if not the only) for health advice. ${ }^{16}$ It is also common practice for African traditional healers to consult the Gods in such cases and this can take the form of incantations and / or divination. While health care based on Western medicine is now increasingly considered the norm in some African communities, several communities in West Africa still rely heavily on African traditional health practices. In, 2010, $\mathrm{WHO}^{14}$ reported that about 70 $80 \%$ of the population in some West African countries depend on African traditional medicine. This was associated to poor access to government-run health facilities, making care by traditional healers or self-medication the preferred health care option for the majority of people in these communities, especially the poor. During the Ebola epidemic, many new cases were traced to contact with a traditional healer, a herbalist or to attendance of a funeral. But the high death rates coupled with the perception that hospitals were places of death, reinforced the lack of compliance with advice to seek early modern medical care. ${ }^{12}$

Also, in some West African communities, traditional healers are more highly regarded than those who promote "unfamiliar" forms of health care. And, while traditional medicine can play a positive role in health care, some ethno-medical beliefs and practices can also have important negative influences on health outcomes and, most importantly, pathogen transmission pathways. For example, studies on the 2014-2015 Ebola outbreak in West Africa revealed that several traditional and spiritual healers falsely claimed the capability to cure Ebola. It is not surprising that several people, including traditional healers themselves, reportedly got infected. By falsely claiming capability to treat and rid patients of Ebola, many traditional healers, lacking proper protective equipment, exposed themselves to infection which they further passed on to their patients. ${ }^{24}$ The media has also reported about several faith healers who prayed for Ebola victims, who not only ended up contracting the disease themselves, but also passing it on to other people. Both Christian and Islamic leaders can, thus, play a significant role in educating their institutional members on the importance of strict adherence to preventive measures for combating the spread of Ebola. Overall, attempts to cure Ebola through traditional and spiritual means highlight the need for increased cooperation between traditional healers, spiritual healers, and trained health personnel. Without fully understanding the etiology, pathogenesis, transmission vehicles, as well as signs and symptoms of the disease, it is difficult for traditional and spiritual healers to distinguish potential Ebola patients from patients with other conditions that they can cure. Furthermore, without proper understanding of the disease, traditional and spiritual healers are often sources of false information and myths to the general public, particularly in remote rural areas where there is limited access to information through electronic and print media. ${ }^{23}$

\section{Compassion}

The EVD epidemic preyed on another deep-seated characteristic of African society: compassion. In West Africa, society is bounded by a culture that stresses on compassionate care for the sick and well as ceremonial processes for their bodies if in the case of death. This is so deep seated that even neighbors feel the need to visit patients at home or in the hospital in order to maintain a good and smooth neighborliness. Some doctors are thought to have been infected with EVD when they rushed, unprotected, to assist patients who has collapsed in waiting or emergency rooms. As several experts have noted, when technical interventions cross purposes with entrenched cultural practices, culture always wins and therefore, health control measures must be embedded in the cultural practices. ${ }^{12}$

\section{Human mobility}

Human movement is considered a particular characteristic of West Africans, with migration rates exceeding those in the rest of the world by more than 7 -fold..$^{25}$ To a large extent, this mobility is driven by poverty as people travel daily looking for work or food. Many extended West African families have relatives living in many different countries around the world. During outbreaks, population mobility creates two significant impediments to control. ${ }^{12}$ First, cross-border contact tracing is difficult and while populations readily cross porous borders, outbreak responders do not. Second, as the situation in one country begins to improve, it attracts patients from neighboring countries seeking available treatment beds, thereby reigniting transmission chains. In other words, as long as one country experiences intense transmission, neighboring countries remain at risk, no matter how strong their own response measures may be. The traditional custom of returning, often over long distances, to a native village to die and be buried near ancestors is another dimension of population movement that carries an especially high transmission risk. A complex suite of sociological and economic factors influence human movement across the landscape and can have critical impacts on outbreak dynamics and the spatial spread of infectious disease. As such, present-day population mobility in West Africa has been an important contributing factor to the explosive nature of the West African Ebola outbreak ${ }^{23}$

\section{Bushmeat consumption}

Bushmeat consumption is a primary mechanism of EVD spillover from wildlife reservoirs to humans. Rapid human migration to urban centers has placed pressure on food production in many parts of West Africa, including access to bush meat, a delicacy and preferred protein source. For example, in Liberia, bush meat is a major source of protein and it is estimated that about three-quarters of the country's population consume bush meat. ${ }^{26}$ Because of apparent food insecurity and poverty, wildlife animals, including bats and primates, are frequently hunted for subsistence and for trade. This amplifies human exposure to zoonotic infections because viruses harbored by these animals can easily be transmitted to humans when their carcasses are consumed as food. Although the mechanism underlying animalto-human transmission of EVD is not entirely clear, most EVD outbreaks, to date, have been traced to a single index case who (or whose family members) had contact with carcasses of bats or primates in impoverished remote African village. ${ }^{23}$

\section{Stigmatization and its implications for outbreak containment efforts}

Stigma can influence the health seeking behavior during an epidemic. This can introduce barriers to outbreak management. Ebola provides an extreme example. Health care workers, critical to outbreak management, have been stigmatized during EVD outbreak; 
rejected by communities, because their communities thought that they are reservoirs for the virus. This has a huge impact in influencing the population's attitude to seek medical help. Fear of stigmatization can also influence the way the disease is reported by victims and their families. Most times infected persons and their families deliberately refused to notify authorities of possible cases of infection because of the potential negative response of their neighbors and community. Ebola survivors can also be heavily stigmatized - many survivors are rejected by their communities..$^{21}$ Stigmatization also goes beyond the immediate family to include relatives of survivors and the deceased. This is also extended to the level of country governance whereby concerns over international response may influence reporting of health information. Health-related stigma has been a prominent feature of the outbreak in West Africa and likely a contributor to the difficulties identified in containing the epidemic. ${ }^{12}$ Health education is one of the many ways to EVD outbreaks. ${ }^{12}$ Along with contacttracing and quarantine in Congo (1995) and Uganda (2000) outbreaks, health education was believed to decrease the effective reproductive rate of Ebola and to reduce the final epidemic size by a factor of 2 . However, as important as it is to develop and share health messages, the messages must also be sensitive to cultural beliefs and practices of the target group.

\section{Environments (physical and social)}

\section{Physical environment that is conducive to EIDs outbreak}

There is a correlation between rainfall and EVD transmission risk. Bausch \& Schwarz ${ }^{27}$ found associations between increased rainfall or humidity and EVD outbreaks. Roads can become impassable with higher levels of rainfall and make it more difficult to seek treatment during outbreaks, and to implement infection control measures, thereby increasing EVD transmission risk. Good roads could decrease risk by improving accessibility to treatment centers during outbreaks. This is one reason that inaccessible remote areas had increased risk of EVD transmission. Moreover, the physical environment in which people live has to be well organized so that no harm comes from it. ${ }^{5}$ Considering the physical environment in West Africa, settlements are generally not well planned and even urban areas are notorious for their rapid development with creation of suburbs and slums that are not conducive for human settlement. The situation in villages, in the majority of the countries of the region, present the worst case scenarios. As Louis Pasteur puts it, the virus "is nothing, the context is all". And in West Africa, the physical context is really "all" with regard to outbreaks and disease occurrence in general.

\section{Social environment}

Some social conditions have an enabling and enhancing effect on human-to-human transmission of EBOV. ${ }^{12}$ War, population growth poverty, and poor health infrastructure, among other social conditions likely contributed to the duration, and size of the EVD epidemic in West Africa. In this region of Africa, population growth has been dramatic, with population densities (people $/ \mathrm{km}^{2}$ ) increasing by around $300 \% .{ }^{12}$ For instance, during the period of 1960-2012 the population density for Guinea was $223 \%$, and $178 \%$, and $275 \%$ respectively for Sierra Leone and Liberia during the period 1961-2013. ${ }^{28}$ Ebola cannot be conquered without understanding Africa's culture, politics and poverty. Wars, social conflicts, corruption, and bribery, cultural traditions, considerably influenced the spread of the 2014-2015 EVD in West Africa. These conditions inhibited the creation of adequate public health facilities in a region where life expectancy at the best of times is as low as 46 years. ${ }^{29}$

\section{Poverty}

Poverty was the most important social determinant of the 20142015 epidemic in West Africa In 2014, WHO's director stated that "poverty is the mother of the current Ebola epidemic" ${ }^{20}$ A 2015 study compared the most economically affected countries by the 2014-2015 EVD outbreak in West Africa (Liberia, Sierra Leone and Guinea) to that of Bill Gates fortune ${ }^{4}$ and the results showed that in 2012, the gross domestic product for the three countries totals 13 billion US dollars while the fortune of Bill Gates was 82 billion dollars, indicating that Bill gates is six times richer than the three nations combined. The social fragility of sub-Saharan Africa has its roots in the extractive nature of its economic institutions and the corruption of the political elites. Until these root causes are seriously addressed it is unlikely that the social determinants that fueled the 2014-2015 Ebola epidemic could be reversed..$^{30}$ Ebola is not only a health emergency, but also a poverty crisis. The Ebola epidemic exacerbated West Africa's poverty crisis through substantial economic loss and social disruption. However, like most neglected tropical diseases (NTD), Ebola is one of the remediable injustices of our times. Addressing the root causes of poverty is the starting point. In West Africa, the epidemic led to food shortages and the neglect of other devastating tropical illnesses like Malaria, Meningitis, and Cholera by public health authorities. ${ }^{31}$

\section{Ethnic conflict, and contemporary wars}

West Africa is one of the world's civil unrest zones. From 1989 to 2004, there has been sustained armed conflict in the region especially in Liberia, Sierra Leone, Guinea, and Côte d'Ivoire. Conflict provides a breeding ground for violence, looting and for the growth of mercenary forces. Also, mass refugee movements as a result of conflicts as well as resettlement camps create a large group of displaced and vulnerable people. These societal disturbances have negatively affected infrastructure, governance, social cohesion, and the mental and physical health and livelihoods of people in the West Africa. Years of civil war and unrest destroyed basic health infrastructures and created a cohort of unemployed youths with little or no education. Road systems, transportation services, and telecommunications are weak in all three affected countries, especially in rural settings. These severely undermines public health infrastructure and service delivery in the region. ${ }^{32}$ Ethnic conflict, political violence and wars that presently shape many parts of world have deep-seated structural causes. Although, the nature of armed conflict has changed substantially over time, the growing number of armed conflicts and wars throughout the world has meant that little attention is given to patterns distress caused by conflict as well as the long-term health impact and psychosocial consequences individuals, communities, or specific ethnic groups in war torn areas. The short or long-term impact assessment on civilian populations of poor countries affected by war have been scarce, and studies on suffering and trauma-related disorders among survivors are only beginning to emerge in the scientific literature. This has led to limited understanding of the relationships among critically important social determinants and the possible health outcomes of political violence. ${ }^{12}$ During epidemics, these environmental weaknesses greatly delayed the transportation of patients to treatment centers samples to laboratories, communication of alerts, reports, calls for help, and public information campaigns. ${ }^{32}$ 


\section{Summary}

\section{Control measures}

Efforts directed at the control diseases in general and EIDs in particular should be informed by SDH. In other words, the way societies are organized in the African region has to be taken into account in dealing with diseases in general and epidemics in particular. ${ }^{33}$ The Commission on Social Determinants of Health ${ }^{5}$ has put it clearly through its overarching recommendations summarized here below:

\section{Improve daily living conditions}

This could be done by putting a major emphasis on early child development and education for girls and boys. Improve living and working conditions and create social protection policies supportive of all, and create conditions for a flourishing older life. Policies to achieve these goals will involve civil society, governments, and global institutions.

\section{Tackle inequitable distribution of power, money, and resources}

In order to address health inequities, it is necessary to address: inequitable conditions of daily living inequities Gender inequities within the society and how it impacts on the health of people within a given community. This will require a public sector that is committed to addressing such inequities and that is equipped, financially and otherwise to take up on such a task. It also requires inclusive governance, that is, legitimacy, space, and support for civil society; an accountable private sector, and for people across society to agree with public interests.

\section{Measure and understand the problem}

Acknowledging that there is a problem is vital for the identification of appropriate action. National governments and international organizations, supported by WHO, should set up health equity surveillance systems for the routine monitoring of health inequity goals, as well as evaluate the health equity impact of policy and action. Creating the organizational space and capacity to act effectively on health inequity requires investment in training of policy-makers, health practitioners, and public understanding of social determinants of health. It also requires a stronger focus on social determinants in public health research.

The focus is then clearly the reduction of the social inequalities. And this cannot be done by the health system alone, it is rather every sector's concern.

\section{Conclusion}

The 2014-2015 Ebola outbreak in West Africa has come to its end with great satisfaction of all stakeholders involved in the fight against that deadly disease. But it is a battle that was won, not the war, as the conditions that led to the outbreak are still generally in place, mainly the social vulnerability of communities. As we have seen in Central Africa, EVD outbreaks will come again (if not again and again) if nothing is done to improve the living conditions of our communities through reduction of social inequalities that are obvious in regions like West Africa, although potentially rich. The 2014-2015 Ebola outbreak in West Africa demonstrated that if scientifically proven methods of combating EIDs are not matched by socio cultural and religious beliefs of target communities, they are likely to be resisted and rendered less effective. There is, thus, a need to investigate and align preventative measures with cultural norms and values of affected communities. Now that the outbreak has been brought under control, there is need to work on the socio-political causes of EVD in particular and EIDs in general.

\section{Acknowledgments}

None.

\section{Conflicts of interest}

Author declares that there is no conflicts of interest.

\section{References}

1. Baize S, Pannetier D, Oestereich L, et al. Emergence of Zaire Ebola virus disease in Guinea - preliminary report. New England Journal of Medicine. 2014;371:1418-1425.

2. CDC. Center for Disease Control and Prevention (2016) 2014 Ebola Outbreak in West Africa - Case Counts.

3. International SOS. Ebola update in Africa. 2014.

4. Henao DE. The Ebola epidemic in West Africa and the social determinants. Rev Peru Med ExpSalud Publica. 2015;32(2):402-403.

5. WHO. Commission on social determinants of health final report. 2018.

6. Frohlich KL. Commentary: What is a population-based intervention? Returning to Geoffrey Rose. International Journal of Epidemiology. 2004;292-1293.

7. WHO. A conceptual framework for action on the social determinants of health. 2007.

8. Kickbusch I. The political determinants of health -10 years on: Public health professionals need to become more politically astute to achieve their goals. BMJ. 2015;350:1-2.

9. Zinszer K, Morrison K, Verma A, et al. Spatial Determinants of Ebola Virus Disease Risk for the West African Epidemic. PLOS Currents Outbreaks. 2017:9.

10. Dowell SF, Mukunu R, Ksiazek TG, et al. Transmission of Ebola hemorrhagic fever: A study of risk factors in family members, Kikwit, Democratic Republic of the Congo, 1995. Commission de Lutte contre les Epidémies à Kikwit. J Infect Dis. 1999;179 Suppl 1:S87-S91.

11. Stanturf JA, Goodrick SL, Warren ML Jr, et al. Social Vulnerability and Ebola Virus Disease in Rural Liberia. PLoS One. 2015;10(9):e0137208.

12. Alexander KA, Sanderson CE, Marathe M, et al. What Factors Might Have Led to the Emergence of Ebola in West Africa? PLoSNegl Trop Dis. 2015;9(6):e0003652.

13. Sabuni LP. Dilemma with the local perception of causes of illnesses in central Africa: muted concept but prevalent in everyday life. Qual Health Res. 2007;17(9):1280-1291.

14. World Health Organization. African traditional medicine day. 2010.

15. Payton G. Families in Liberia are paying bribes for false certificates over Ebola deaths. 2015.

16. Houéto D. Community Empowerment for the Control of Child Malaria in Benin. European University Editions: 2010.

17. Lee R, Vaughan MM. Death and dying in the history of Africa since 1800. The J of Afr Hist. 2008;49(03):341-359.

18. Anstey S. Wildlife Utilisation in Liberia WWF. FDA Wildlife Survey Report. 1991. 
19. Nielsen Carie F, Kidd S, Sillah AR, et al. Improving burial practices and cemetery management during an Ebola virus disease epidemic-Sierra Leone. MMWR. 2014;64(1):20.

20. Chan M. Ebola Virus Disease in West Africa-No Early End to the Outbreak. New England Journal of Medicine. 2014;371:1183-1185.

21. Hewlett Barry S, Amola Richard P. Cultural contexts of Ebola in northern Uganda. Emerg Infect Dis. 2003;9(10):1242.

22. Paye-Layleh J. Liberia eases up on cremation order for Ebola victims 2014.

23. Manguvo A, Mafuvadze B. The impact of traditional and religious practices on the spread of Ebola in West Africa: time for a strategic shift. Pan Afr Med J. 2015;22(Supp 1):9.

24. Umeora O, Emma-Echiegu N, Umeora MC, et al. Ebola viral disease in Nigeria: the panic and cultural threat. Af J of Med and Health Sci. 2014;13(1):1-5.

25. ECOWSA-SWAC/OECD. Atlas on Regional Integration in West Africa Population Series. 2006.

26. Mbete RA, Banga-Mboko H, Racey P, et al. Household bushmeat consumption in Brazzaville, the Republic of the Congo. Tropical Conservation Science. 2011;4:187-202.
27. Bausch DG, Schwarz L. Outbreak of Ebola virus disease in Guinea: where ecology meets economy. PLoS neglected tropical diseases. 2014;8:e3056.

28. The World Bank Data. 2014.

29. Ikuomola FI. The Ebola Virus and West Africa: Medical and Sociocultural Aspects. iUniverse; 2015:185.

30. Roca A, Afolabi MO, Saidu Y, et al. Ebola: A holistic approach is required to achieve effective management and control. $J$ Allergy Clin Immunol. 2015;135(4):856-867.

31. Troncoso A. Ebola outbreak in West Africa: a neglected tropical disease. Asian Pac J Trop Biomed. 2015;5(4):255-259.

32. Olugasa BO, Dogba JB, Ogunro B, et al. The rubber plantation environment and Lassa fever epidemics in Liberia, 2008-2012: A spatial regression. Spatial and Spatio-temporal Epidemiology. 2014;11:1-174.

33. Chigudu S. Politics and epidemics. BMJ Talk Medicine: 2019. 